\title{
El uso de benzodiacepinas estaría asociado a la enfermedad de Alzheimer: estudio caso-control
}

\author{
Benzodiazepine use would be associated with Alzheimer's disease: case-control study
}

\section{Objetivos}

Investigar la relación entre el uso de benzodiacepinas durante 5 años o más y el riesgo de desarrollar enfermedad de Alzheimer, teniendo en cuenta la dosis y la vida media de la droga.

\section{Diseño, lugar y pacientes}

Estudio caso-control de pacientes mayores de 66 años de la comunidad de Quebec (Canadá). Se tomaron datos desde el 1 enero de 2000 hasta diciembre de 2009. Se incluyeron 1796 pacientes como casos, con demencia tipo Alzheimer (según criterios de la clasificación internacional CIE-10), sin evidencia de tratamiento para demencia previo y 7184 controles apareados por sexo, grupo etario y tiempo de seguimiento. Ambos grupos tenían un seguimiento de al menos 6 años. No se incluyó en el análisis el consumo de benzodiacepinas en un tiempo menor de 5 años de la fecha de diagnóstico a fin de evitar un sesgo de causalidad inversa.

\section{Evaluación de los factores de riesgo}

Se midió la prevalencia e intensidad de exposición a las benzo-
Billioti de Gage S y col. BMJ 2014; 349:1-10 diacepinas categorizándolas según su vida media corta o de larga duración considerando como larga duración cuando se trataba de un consumo mixto.

La intensidad de exposición se midió a través de la densidad acumulada de exposición a benzodiacepinas en cada paciente durante el período de estudio, trasladando el análisis final a un consumo promedio por día por fármaco. Se consideraron tres categorías de dosis acumuladas: menos de tres meses de consumo, entre tres y seis meses, y más de seis meses.

\section{Medición de resultados principales}

Los principales resultados se resumen en la tabla 1. La exposición acumulada de benzodiacepinas por un período mayor a seis meses fue más común entre las personas con Alzheimer $(32,9 \%)$ que en los controles $(21,8 \%)$, siendo más fuerte la asociación con la enfermedad para los fármacos de vida media prolongada que para los de vida media corta. No se encontró asociación significativa para la ansiedad $(p=0,48)$, depresión $(p=0,75)$, o insomnio $(p=0,99)$.

Tabla 1. Riesgo de enfermedad de Alzheimer asociada con el uso de benzodiacepinas

\begin{tabular}{|c|c|c|c|c|}
\hline Utilización de benzodiacepinas & $\begin{array}{l}\text { Casos }(n, \%) \\
\quad N: 1796\end{array}$ & $\begin{array}{c}\text { Controles (\%) } \\
\text { N: } 7184\end{array}$ & $\begin{array}{l}\mathrm{OR}^{\perp} \text { univariado } \\
\text { (IC 95\%) }\end{array}$ & $\begin{array}{l}\mathrm{OR}^{\triangle} \text { multivariado } \\
\text { (IC } 95 \%)^{*}\end{array}$ \\
\hline Prevalencia de uso & $894(49,8)$ & $2.873(40,0)$ & $1,52(1,37$ a 1,69$)$ & $1,51(1,36$ a 1,69$)$ \\
\hline \multicolumn{5}{|l|}{ Densidad de exposición según período de uso } \\
\hline$<3$ meses & $234(13 \%)$ & $1.051(14,6 \%)$ & $1,08(0,92 \mathrm{a} 1,27)$ & $1,09(0,92$ a 1,28$)$ \\
\hline 3 a 6 meses & $79(3,9 \%)$ & $257(3,6 \%)$ & $1,33(1,01$ a 1,75$)$ & $1,32(1,01$ a 1,74$)$ \\
\hline$>6$ meses & $590(32,9 \%)$ & $1.565(21,8 \%)$ & $1,85(1,63$ a 2,09$)$ & $1,84(1,62$ a 2,08$)$ \\
\hline \multicolumn{5}{|l|}{ Exposición según vida media } \\
\hline Vida media corta ( $\leq 20$ horas) & $585(32,6 \%)$ & $1.996(27,8 \%)$ & $1,43(1,27$ a 1,61$)$ & $1,43(1,27$ a 1,61$)$ \\
\hline Vida media larga (> 20 horas) & $309(17,2 \%)$ & $872(12,2 \%)$ & $1,72(1,48$ a 1,99$)$ & $1,70(1,46$ a 1,98$)$ \\
\hline
\end{tabular}

$\triangle \mathrm{OR}$ : odds ratio *Análisis ajustado por hipertensión arterial, infarto agudo de miocardio, accidente cerebrovascular, hipercolesterolemia, diabetes mellitus, consumo de antiagregantes o anticoagulantes, ansiedad, depresión, insomnio y otras comorbilidades (enfermedad pulmonar obstructiva, enfermedad del colágeno, enfermedad. hepática, hemiplejía o paraplejía, insuficiencia renal, cáncer, inmunodeficiencia adquirida).

\section{Conclusión}

Este estudio mostró una asociación entre el uso crónico de benzodiacepinas y el riesgo de Alzheimer. El riesgo se incrementa aún más con la densidad de exposición y el uso de ben- zodiacepinas de vida media larga. El ajuste por síntomas como la ansiedad, que pueden ser considerados pródromos de la demencia, no modificaron significativamente los resultados.

\section{Comentario}

Los efectos perjudiciales de las benzodiacepinas sobre la memoria están bien documentados, pero no se conoce un mecanismo fisiopatológico que explique el aumento del riesgo de desarrollar demencia ${ }^{1,2}$. Una revisión sistemática reciente reveló que el uso de benzodiacepinas induce tanto deterioro cognitivo leve no amnésico y amnésico y una progresión más rápida de ésta última forma de deterioro a demencia tipo Alzheimer $^{3}$. Bajo un supuesto de causalidad, la hipótesis más factible sería la disminución en la reserva cognitiva inducida por el uso crónico de benzodiacepinas, lo que podría reducir la capacidad para afrontar las lesiones cerebrales en una fase temprana del deterioro, sin posibilidad de crear nuevas conexiones neuronales ${ }^{4,5}$. Las benzodiacepinas son herramientas de gran utilidad para el manejo de los trastornos de ansiedad y del sueño. Pero tal como lo indican las normas internacionales no deberían ser usadas por más de 3 meses. El resultado de este estudio refuerza la sospecha sobre el riesgo elevado de desarrollar demencia tipo Alzheimer entre los usuarios crónicos de benzodiacepinas, y proporciona un argumento a favor de evaluar cuidadosamente la indicación de estas drogas en ancianos. Dado que hasta el momento no existe un tratamiento curativo o preventivo para la enfermedad de Alzheimer, este factor de riesgo modificable debe ser tenido en cuenta a la hora de indicar una benzodiacepina en ancianos, que además se asocia a un riesgo elevado de caídas y fracturas, otra comorbilidad de gran impacto en la salud pública.

María Elena Guajardo [ Servicio Clínica Médica, Sección Geriatría del Hospital Italiano de Buenos Aires. maria.guajardo@ hospitalitaliano.org.ar ]

Guajardo M. El uso de Benzodiazepinas estaría asociado a la enfermedad de Alzheimer: estudio caso-control. Evid Actual Pract Ambul. 2015;18(4):117. Oct-Dic. Comentado de: Billioti de Gage S, y col. Benzodiazepine use and risk of Alzheimer's disease: case-control study. BMJ. 2014; 349(g5205). PMID: 25208536.

\section{Referencias:}

1. Ghoneim MM, y Col. Benzodiazepines and human memory: a review. Anesthesiology 1990; 72: 926-938

2. Curran HV. Tranquilising memories: a review of the effects of benzodiazepines on human memory. Biol Psychol 1986; 23:45-58.

3. Tannenbaum C, y Col. A systematic review of amnestic and non-amnestic mild cognitive impaiment induced by anticholinergic, antihistamine, GABAergic and opioid drugs. Drugs Aging 2012;58:1985-1992

4. Petersen RC, y Col. Current concepts in mild cognitive impaiment. Arch Neurol 2001; 58: 1985-1992.

5. Busse A, y Col. Mild cognitive impaiment: long -term course of four clinical subtypes. Neurology 2006; 67: 2176-2185. 